\title{
AMBIENTE DO VISUAL MERCHANDISING EM LOJAS DE VAREJO DE SANTA MARIA
}

\author{
Maritielle Carolaine Brasil Ayres ${ }^{1}$, Lucas Gonçalves \\ Pagnossin², Ana Margarete Rodrigues Martins Ferreira ${ }^{3}$
}

\begin{abstract}
RESUMO: A utilização do merchandising faz parte das organizações a um longo tempo. Com esta ferramenta as empresas buscam atingir e transmitir informações aos seus clientes, com o intuito de manterem-se competitivas no mercado. 0 objetivo deste trabalho foi mapear os índices de utilização dos tipos de abordagens do visual merchandising em Santa Maria-RS. O procedimento metodológico utilizado é uma pesquisa exploratória. Foi realizado um levantamento em sete segmentos de lojas de varejo da cidade com o objetivo de identificar os tipos dos elementos de visual Merchandising utilizados. As lojas pesquisadas utilizam a abordagens via etiquetas de preços, sinalização de vitrine ou entrada, banners, balcões de demonstração e adesivos. Assim, tem-se um panorama atualizado quanto às práticas adotadas pelas lojas selecionadas como amostra neste trabalho.
\end{abstract}

Palavras-chave: Merchandising. Competitividade. Pesquisa Survey.

Submissão: 14/05/2019

Aceite: $27 / 05 / 2019$

DOI: $10.47591 / 2674-9203.2019 v 1 n 1 . a r t 3-25-34$

\section{(c) (1) (9)}

Este trabalho está licenciado com uma Licença Creative Commons Atribuição-NãoComercial 4.0 Internacional.

\footnotetext{
${ }^{1}$ E-mail: krol.brasil4@gmail.com

${ }^{2}$ E-mail: lucaspagnossin@gmail.com

${ }^{3}$ E-mail: anamartinsferreira@gmail.com
} 


\section{INTRODUÇÃO}

Baseado nos conceitos estudados, este trabalho buscou realizar uma pesquisa exploratória do tipo levantamento direto, com o propósito de obter informações sobre o comportamento em relação a abordagens de visual merchandising por uma amostra de lojas de varejo na cidade de Santa Maria-RS, considerando principalmente aquelas que possuem mais de uma filial, com a finalidade de excluir empresas consideradas pequenas. Desta forma, o presente estudo aborda um levantamento sobre práticas de merchandising visual em 24 empresas localizadas em um espaço comercial localizadas no centro da cidade. Este tema está relacionado ao problema de pesquisa que originou este trabalho e que pode ser descrito da seguinte maneira: quais os elementos básicos de ambiente abordados pelo Visual Merchandising mais explorados para atrair potenciais consumidores nas lojas de varejo com mais de um ponto de venda situadas no centro de Santa Maria?

Como objetivo geral, este trabalho busca identificar o percentual de utilização dos elementos de imagem e ambiente do Visual Merchandising para atrair os clientes nas lojas que possuem mais de uma filial no Centro da cidade de Santa Maria. Considerando a comunicação visual, iluminação, cores, música e aromas (BLESSA, 2005 apud MEMÓRIA, 2010). Como objetivos específicos teve: proceder com a delimitação e a seleção de uma amostra para uma pesquisa no centro de Santa Maria; identificar e apresentar os resultados da pesquisa evidenciando o percentual dos elementos utilizados pelas lojas.

Para tanto, este texto apresenta na primeira seção a definição do tema e do problema de pesquisa, bem como, as questões de pesquisa e os objetivos. Na segunda seção é abordada uma revisão teórica, seguida por resultados de outros trabalhos já realizados e relacionados com merchandising. Na terceira seção são apresentados os procedimentos metodológicos, abordando o cenário, a classificação e as etapas da pesquisa. Sequencialmente, na quarta são apresentados os resultados e, por fim, na quinta seção será apresentada a conclusão do trabalho.

\section{MERCHANDISING}

Atualmente as empresas estão inseridas em um cenário competitivo, instável e dinâmico, onde o avanço tecnológico, a globalização e ainda as mudanças na legislação e na economia influenciam diretamente nas estratégias industriais nos diferentes setores. Neste cenário, as organizações para manterem-se competitivas utilizam ferramentas, técnicas e metodologias, dentre outras práticas de gestão para atingirem seus clientes (BATISTA, 2006).

Devido à grande necessidade de alcançar índices melhores de vendas diante da concorrência, as tendências comportamentais do consumidor e de produtos similares, as técnicas de Visual Merchandising vêm ficando cada vez mais sofisticadas nos últimos anos. Merchandising é definida como um conceito de negócio que é uma das formas de comunicação de uma marca, ou seja, a visual. Esta técnica possui como propósito expor todo e qualquer produto, atrair clientes e como resultado ascender às vendas (DEMETRESCO, 2015). 
O Visual merchandising é uma estratégia de varejo que trabalha o ambiente do ponto de venda, e cria uma identidade da loja ou da marca a ser trabalhada, também personaliza o ambiente através do design, do layout e da disposição dos produtos, impulsionando e influenciando nas decisões de compra dos clientes. (SEBRAE, 2019).

Sendo assim, o merchandising serve para os consumidores receberem informações relevantes, simplificando o processo de compra. Além de informar, que é sua função básica, esta ferramenta tem como funções também, lembrar aos consumidores as marcas e encorajar os mesmos a comprarem os itens expostos (SHIMP, 2012). O citado autor aponta que os materiais de merchandising talvez desempenhem um papel principal em influenciar as compras não planejadas. Em um estudo realizado do comportamento de compra de consumidores, verificou que grande parte das compras realizadas são caracterizadas desta forma, ou seja, o cliente acaba decidindo comprar o produto dentro da loja, mesmo que sua intenção seja comprar somente um determinado produto.

Nesta linha de pensamento, Memória (2010) realizou uma pesquisa com uma amostra de 200 consumidores em dois supermercados, sendo analisados 100 consumidores em cada estabelecimento, para analisar o comportamento no momento das compras e como o merchandising pode influenciar na compra por impulso. Neste contexto, verificou-se que o consumidor realiza várias compras, que não foram planejadas, antes de chegar ao ponto de venda. Sendo assim, os produtos bem expostos, com um visual agradável ao cliente e em promoção possuem maiores chances de serem adquiridos.

A fim de investigar o efeito dos fatores de merchandising em vendas de varejo, Feijó e Botelho (2012) realizaram um estudo em dois grupos de lojas, onde um seria implantado o merchandising e o outro permaneceria inalterado. Como resultados encontraram que os grupos que aplicaram os fatores de merchandising obtiveram aumento na receita, ou seja, esse acréscimo pode ser um indicador que a ferramenta exerce influência no comportamento do consumidor, levando-o a consumir mais.

Silva e Pinheiro (2006) realizaram uma pesquisa para verificar como os clientes sentem-se no ambiente de uma loja que usa estes instrumentos para atrair clientes e outra que não utiliza nenhum tipo de atração. Como conclusão, destaca que na loja que melhorou o ambiente para as experiências dos clientes o mesmo sente-se mais confortável para realizar a compra e sempre adquire produtos que não estavam em sua lista de compras, apenas pela exposição destes no local adequado. Segundo este mesmo estudo, o vitrinismo é a ferramenta de atração mais eficaz para atingir atenção dos consumidores. Pois observa-se que sempre tem consumidores, que mesmo que não estejam em compras, observam as vitrines e acabam entrando e fazendo compras, sendo atraídas por elas.

Oliveira e Santos (2011), realizaram uma pesquisa bibliográfica exploratória com os vários elementos que constituem o merchandising e a importância de cada um deles no processo de persuasão e produção de estímulo para as compras, destacando o papel de cada uma, seus objetivos e conquistas. Neste mesmo tipo de pesquisa, Almeida e Marques (2012), afirmam que o merchandising desperta os desejos e emoções nos clientes que geram o consumo. Afirmam também que todo o ambiente de compra da loja, ambiente externo, tipo de fachada, placa de 
identificação, vitrine, layout, iluminações, cores, aroma e som precisam ser previamente planejados para que o consumidor receba a informação que a loja quer passar.

Conforme SEBRAE (2019), esta ferramenta incita os colaboradores responsáveis por esta função, estar sempre inovando, para cada vez atrair mais público:

A principal função do Visual Merchandising é ser uma ferramenta criativa e movimentadora para as vendas, ela também orienta os seus colaboradores para pensarem constantemente de forma cada vez mais comercial, fazendo com que eles estejam sempre pensando no impacto que podem causar no seu público alvo.

Por fim, vale ressaltar que o merchandising é uma ferramenta de grande relevância, fato comprovado pelos diversos estudos já realizados por autores ao longo dos anos abordando-o de diferentes modos, como por exemplo, aspectos sobre as vendas em varejos de esportes e roupas de moda-íntima (BAKER, J et al, 2002; KERFOOT; DAVIES;WARD, 2003; DAVIES, J. B.; WARD, 2005; GABAY et al., 2010; FEIJÓ; BOTELHO, 2012; LAW; WONG; YIP, 2012; CARENYS; SALES, 2012).

\section{MÉTODO}

A seção de procedimentos metodológicos visa apresentar o cenário, o método e as etapas de pesquisa.

0 presente estudo realizou um levantamento de dados através de uma pesquisa exploratória, afim de, identificar e investigar o comportamento de pontos de vendas localizadas no centro de Santa Maria. Neste contexto, foram selecionadas lojas de forma intencional e não probabilística. Esta amostra foi selecionada entre as lojas que tinham mais de uma filial e que uma destas fosse localizada na área central da cidade, portanto esta amostra foi composta 24 lojas que possuem mais de um ponto de venda na cidade ou fora de Santa Maria.

Esta pesquisa é apontada quanto à natureza como aplicada. Isto é dito por esta pesquisa estar voltada a adquirir conhecimentos com o intuito de aplicação em um contexto real (GIL, 2010). Quanto aos objetivos é pesquisa exploratória, pois visa tornar o problema de pesquisa mais explícito (GIL, 2010). Quanto aos procedimentos técnicos é considerado do tipo levantamento, visto que, descreve as características de uma determinada amostra, com o objetivo de obter conhecimentos sobre um universo pesquisado (MIGUEL, 2010). Quanto à abordagem de pesquisa é considerada quantitativa, pois esse tipo apresenta uma quantificação da coleta de informações e tratamento de dados (PINHEIRO, 2010). Quanto ao método de pesquisa: indutivo, porque partirá de características individuais das lojas para concluir as questões relacionadas às lojas de varejo com mais de um ponto de venda situadas no centro de Santa Maria, Rio Grande do Sul. (GIL, 2008).

A primeira etapa do projeto foi a abordagem da definição do problema de pesquisa e os objetivos. A segunda, o referencial teórico, que implica em revisar a literatura buscando informações sobre definições e aplicações correlacionadas com o problema de pesquisa. Posteriormente, na terceira seção, foi realizada a 
caracterização da amostra, elaboração do método de coleta de dados e do instrumento de pesquisa. Na quarta etapa, foi realizada a coleta a partir da aplicação dos instrumentos de pesquisa e análises. Por fim, na quinta etapa, foi concluído o trabalho a partir dos dados obtidos.

A coleta de dados foi feita nos meses de outubro e novembro de 2018, pelos autores do trabalho. Durante coleta de dados, foram listadas em primeiro momento, as lojas que compõe a amostragem. Sequencialmente, foi realizada a observação das mesmas em relação às características apontadas na ferramenta de coleta de dados (Quadro 1). Neste contexto, a ferramenta apresenta 8 campos conforme a ferramenta desenvolvida sobre a utilização ou não de técnicas e ações de merchandising nos pontos de venda (BLESSA, 2005 apud MEMÓRIA, 2010). Os conceitos de cada campo são apresentados no Quadro 2.

Por fim, a coleta foi feita por observação sistemática, onde foram somente considerados os aspectos previamente definidos no instrumento de coleta de dados nas lojas de varejo com mais de um ponto de venda situadas no centro de Santa Maria, Rio Grande do Sul. Utilizando uma matriz sobre a utilização ou não das características que foram observadas.

Quadro 1- Demonstrativo das características observadas nas lojas selecionadas do centro de Santa Maria-RS.

\begin{tabular}{|c|c|c|c|c|c|c|c|c|c|c|c|c|c|c|c|c|c|c|c|c|c|c|c|c|}
\hline \multirow[b]{2}{*}{ Características } & \multicolumn{24}{|c|}{ Utilização das características pelas lojas. } \\
\hline & -1 & $N$ & $m$ & + & เ & 6 & $\wedge$ & $\infty$ & $a$ & 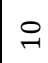 & $\exists$ & 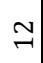 & $\stackrel{m}{\rightarrow}$ & $\stackrel{\leftrightarrows}{\leftrightarrows}$ & $\stackrel{2}{\sim}$ & 0 & $\approx$ & $\stackrel{\infty}{\sim}$ & $\stackrel{\sigma}{\rightarrow}$ & 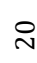 & $\vec{\sim}$ & 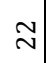 & $\approx$ & $\stackrel{\mathrm{N}}{4}$ \\
\hline Adesivos & & & & & & & & & & & & & & & & & & & & & & & & \\
\hline $\begin{array}{l}\text { Balcão para } \\
\text { demonstração }\end{array}$ & & & & & & & & & & & & & & & & & & & & & & & & \\
\hline Bandeirolas & & & & & & & & & & & & & & & & & & & & & & & & \\
\hline Banner & & & & & & & & & & & & & & & & & & & & & & & & \\
\hline Etiquetas de preço & & & & & & & & & & & & & & & & & & & & & & & & \\
\hline Infláveis & & & & & & & & & & & & & & & & & & & & & & & & \\
\hline Móbile & & & & & & & & & & & & & & & & & & & & & & & & \\
\hline $\begin{array}{l}\text { Sinalização de vitrine } \\
\text { ou entrada }\end{array}$ & & & & & & & & & & & & & & & & & & & & & & & & \\
\hline
\end{tabular}

Quadro 2 - Tipos de merchandising analisado

\begin{tabular}{|l|l|}
\hline Adesivos & $\begin{array}{l}\text { Materiais que aderem em superfícies, inclusive no chão das } \\
\text { lojas. }\end{array}$ \\
\hline Balcão para demonstração & $\begin{array}{l}\text { Estande pequeno onde fica uma demonstradora que divulga o } \\
\text { produto, esclarecendo suas características e benefícios }\end{array}$ \\
\hline Bandeirolas & $\begin{array}{l}\text { Bandeirinhas suspensas por um cordão ou barbante no teto } \\
\text { dos supermercados. No entanto, parece pouco utilizada } \\
\text { atualmente }\end{array}$ \\
\hline Banner & $\begin{array}{l}\text { Sinalizadores feito em plástico ou tecido, nos mais variados } \\
\text { tamanhos, que sinalizam as características de um produto, } \\
\text { tendo a vantagem de possuir baixo custo }\end{array}$ \\
\hline Etiquetas de preço & $\begin{array}{l}\text { Materiais obrigatórios de uso da loja, onde se encontram } \\
\text { afixados os preços dos produtos, mas que, como forma de } \\
\text { destaque, podem vir em formato maior ou colorido }\end{array}$ \\
\hline
\end{tabular}




\begin{tabular}{|l|l|} 
Infláveis & $\begin{array}{l}\text { Displays, geralmente de plástico, que se apresentam nas } \\
\text { formas das embalagens de produtos, balões infláveis ou nas } \\
\text { mais variadas formas }\end{array}$ \\
\hline Móbile & $\begin{array}{l}\text { São peças que contêm apenas a imagem do produto e a marca, } \\
\text { que ficam suspensas por fios em uma gôndola ou no teto. } \\
\text { Muito usado em farmácias e supermercados }\end{array}$ \\
\hline Sinalização de vitrine ou entrada & $\begin{array}{l}\text { Qualquer tipo de material impresso afixado na vitrine, que } \\
\text { informa sobre produtos ou promoções }\end{array}$ \\
\hline
\end{tabular}

Fonte: Adaptada de BLESSA, 2005 apud MEMÓRIA, 2010.

Na Figura 1 é apresentado o tipo de comércio utilizado na pesquisa, considerando-se que no local se encontram maior número de lojas de roupas e eletrônicos e menor número de livrarias e lojas de brinquedos.

Figura 1- Caracterização da amostra considerando segmento de comércio

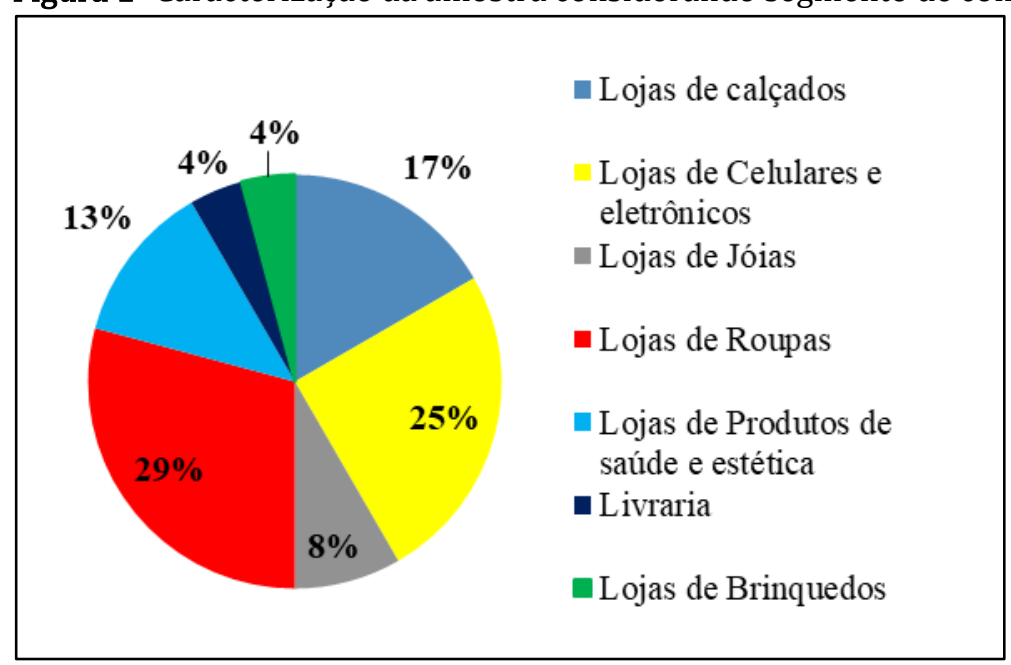

Fonte: dados da pesquisa.

\section{ANÁLISE E DISCUSSÃO DOS RESULTADOS}

Em uma análise geral são apresentados no quadro 3, a porcentagem das lojas pesquisadas que utilizam os tipos de merchandising, observa-se em linhas gerais que a maioria delas utiliza etiquetas de preço e nenhuma delas utiliza infláveis. Todos estes itens serão discutidos separadamente.

Quadro 3- Porcentagem de lojas que utilizam cada tipo de merchandising

\begin{tabular}{|l|c|c|}
\hline Tipo de abordagem & Possui (\%) & Não possui (\%) \\
\hline Adesivos & 37 & 63 \\
\hline Balcões de demonstração & 42 & 58 \\
\hline Bandeirolas & 8 & 92 \\
\hline Banner & 42 & 58 \\
\hline Etiquetas de preços & 75 & 25 \\
\hline Infláveis & 0 & 100 \\
\hline Mobile & 21 & 79 \\
\hline Sinalização de vitrine ou entrada & 54 & 46 \\
\hline
\end{tabular}


Na Figura 2 tem-se a porcentagem da utilização de adesivos pelas lojas pesquisadas, nota-se que não são utilizados por uma grande maioria das lojas, e sim em apenas $37 \%$ delas, mostrando que não é considerada eficaz pelos comerciantes.

Figura 2- Utilização de adesivos nas lojas pesquisadas.

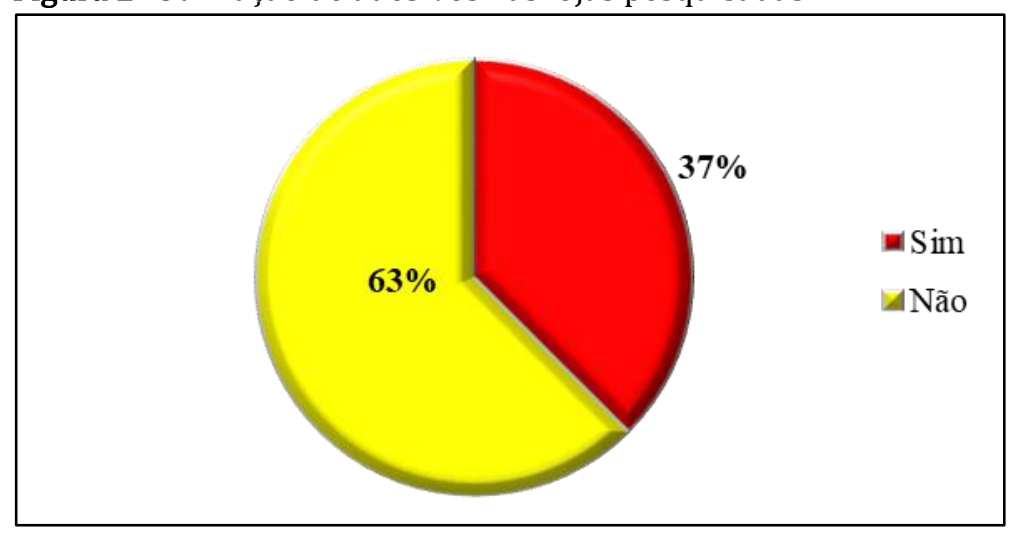

Na Figura 3 se observa que balcões de demonstração não são utilizados em $58 \%$ das lojas, pelo resultado pode-se inferir que esta modalidade de merchandising, não deve atrair muito as pessoas que visitam estas lojas e que os colaboradores não consideram esta característica muito importante. Diferentemente ao observado em lojas populares e ou supermercados, que a maioria deles considera esta modalidade pois a maioria dos consumidores compram o que não estava previsto quando se deparam com produtos em balcões de demonstração (MEMÓRIA, 2010).

Figura 3 - Utilização de balcões de demonstração

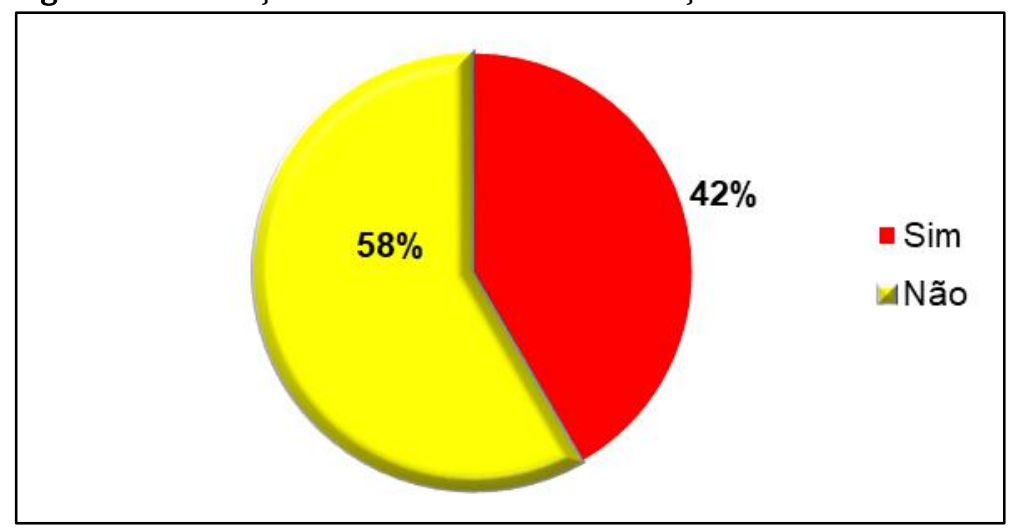

Pôde-se observar que as bandeirolas e banneres não são as preferidas dos lojistas, pois foram encontradas em apenas $8 \%$ das lojas pesquisadas e que estas são ainda os que causam menores interesses dos lojistas para chamar atenção de seus clientes.

Observou-se também que a etiqueta de preços é visível em $75 \%$ das lojas, (Figura 4) apesar do Decreto nº 5.903/2006, em seu Art. 4o determinar que: "Os preços dos produtos e serviços expostos à venda devem ficar sempre visíveis aos consumidores enquanto o estabelecimento estiver aberto ao público" e o Código de Defesa do Consumidor também determina em seu Art. 6ำ que "(...) III - a informação adequada e clara sobre os diferentes produtos e serviços, com especificação correta 
de quantidade, características, composição, qualidade, tributos incidentes e preço, bem como sobre os riscos que apresentem". Observou-se, porém que m $25 \%$ das lojas pesquisadas esta não é uma prática recorrente, onde os lojistas preferem dar as informações de preços diretamente pelo vendedor.

Figura 4 - Utilização de etiquetas de preços

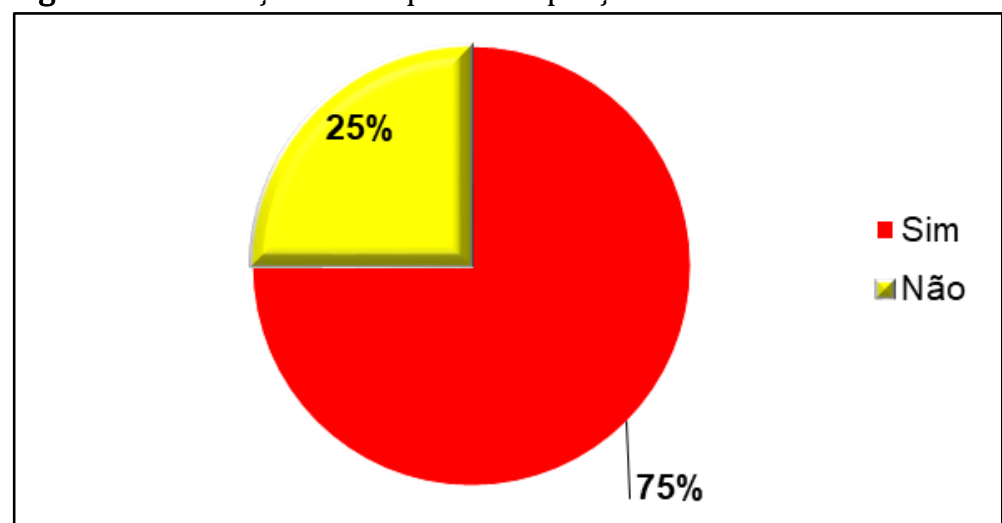

Em nenhuma das lojas pesquisadas observou-se a utilização de infláveis no entanto $21 \%$ delas utilizam móbiles como sinalizador de seus produtos (Figuras 5).

Figura 5 - Utilização de mobile

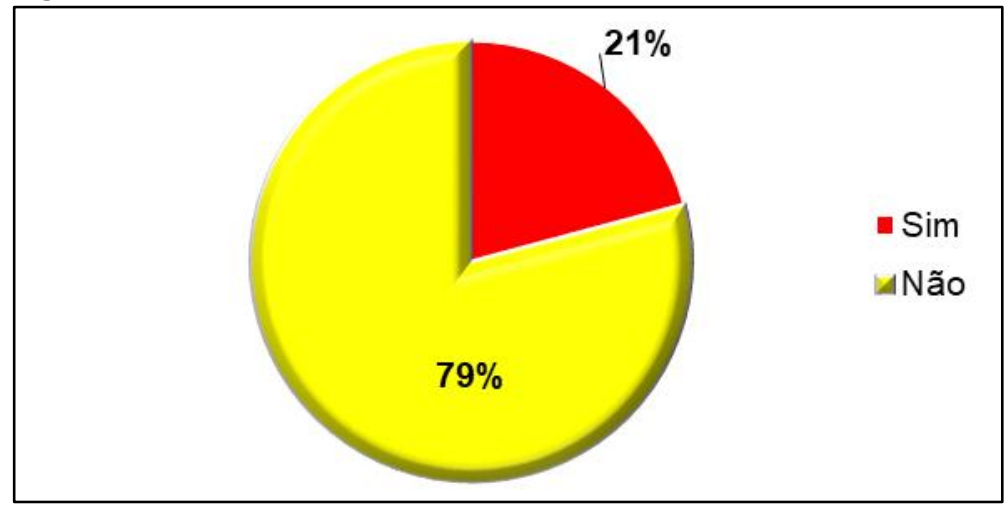

Cinquenta e quatro por cento das lojas pesquisadas possuem vitrine ou uma entrada com produtos (Figura 6) que chamam a atenção de clientes para preços ou promoções. Considera-se que uma vitrine atraente é estratégica para aumentar as vendas, assim o lojista tem que ter o cuidado nos espaços de exposição para cativar seu cliente (SEBRAE, 2016).

Figura 6 - Sinalização de vitrine ou entrada 


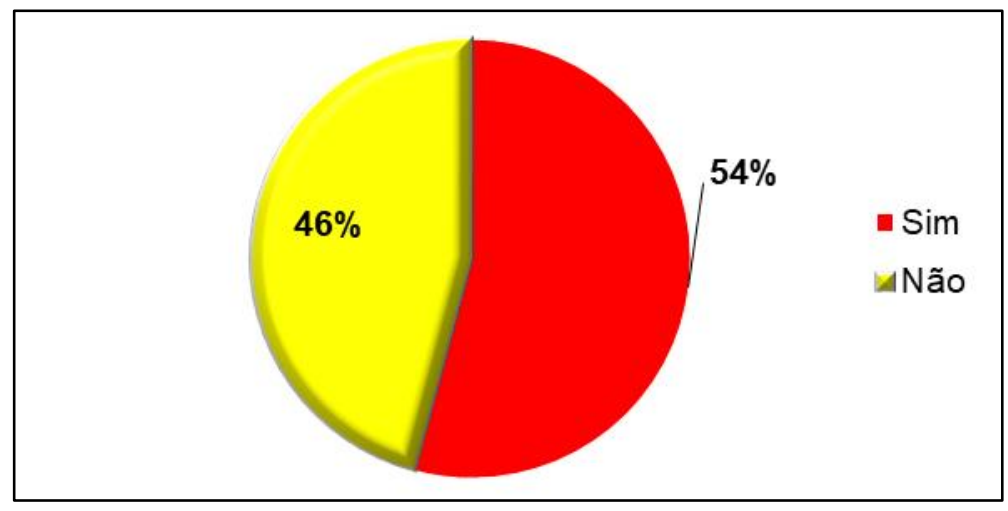

\section{CONCLUSÕES OU CONSIDERAÇÕES FINAIS}

Neste trabalho foram apresentados resultados de uma pesquisa que busca levantar quais os tipos de abordagens de visual merchandising que as lojas de varejo com mais de uma filial, localizadas no centro de Santa Maria - RS utilizam para atrair seus clientes para compras. .

Como resultados obteve-se que as lojas utilizam a abordagens via etiquetas de preços, sinalização de vitrine ou entrada, banners, balcões de demonstração e adesivos. Um resultado a destacar é que todas as lojas de calçados e de celulares e eletrônicos possuem etiquetas de preços à vista na parte frontal de sua loja, assim como todas as lojas desse segundo segmento possuem banner e sinalização de vitrine ou entrada.

Por fim, vale ressaltar que o resultado dessa pesquisa apresenta a delimitação de apenas um espaço comercial localizado na área central na cidade de Santa Maria, não podendo ser generalizado. Recomenda-se em estudos futuros a ampliação deste espaço, para se ter uma maior certeza, para se qualificar os espaços de visual merchandising utilizados pelas lojas nesta cidade.

\section{REFERÊNCIAS}

ALMEIDA, J. S. C. ; MARQUES, N. S. Merchandising no Ponto de vendas: uma ferramenta estratégica. In: VII Convibra. 7., 2012, Rio de Janeiro. Anais.... Rio de Janeiro: Convibra, 2012.. Disponível em: <http://www.convibra.com.br/upload/paper/2012/37/2012_37_4433.pdf> Acesso em: 14 de nov. 2017.

BAKER, J et al. The influence of multiple store environment cues on perceived merchandise value and patronage intentions. Journal of Retailing, v. 66, n. 2, p. 120-141, 2002.

BATISTA, Emerson de O. Sistema de Informação: 0 uso consciente da tecnologia para o gerenciamento. Ed. Saraiva, 2006.

BLESSA, R. Merchandising no ponto de venda. 3.ed. São Paulo: Atlas, 2005

CARENYS, J., SALES X., (2012) "Tailoring performance management systems: a sports merchandiser's case", Sport, Business and Management: An International Journal, v. 2 n. 2, p.115126, 2012.

DAVIES, J. B.; WARD, P. "Exploring the connections between visual merchandising and retail branding: An application of facet theory", International Journal of Retail \& Distribution Management, v. 33 n. 7, p.505-513, 2005.

DEMETRESCO, S. Vitrinas e exposições : arte e técnica do visual merchansiding. 1. ed. São Paulo: Érica, 2014. 
FEIJÓ, F. R.; BOTELHO, D. Efeito dos fatores de merchandising nas vendas do varejo. Revista de Administração de Empresas. v. 52, n.6, 2012

GABAY, G et al. "Creating technology-based merchandising ideas for hair coloring through weak signals, concept optimization and mind-set segmentation", Journal of Consumer Marketing, v. $27 \mathrm{n}$. 3, p. 211-223, 2010.

GIL, A. C. Como elaborar projetos de pesquisa. 5.ed. São Paulo: Atlas, 2010.

GIL, A. C. Quadro 2 - Caracterização dos tipos de merchandising analisado. 6.ed. São Paulo: Atlas, 2008.

KERFOOT S. DAVIES, B., WARD, P. "Visual merchandising and the creation of discernible retail brands", International Journal of Retail \& Distribution Management, v. 31 n. 3, p.143-152, 2003.

LAW, D.; Wong, C.; YIP, J. How does visual merchandising affect consumer affective response? : An intimate apparel experience", European Journal of Marketing, Vol. 46 n. 1/2, p.112-133, 2012)

MEMÓRIA, P.R.F . O Merchandising como ferramenta de estratégia para vendas no varejo. Revista Eletrônica da Faculdade Metodista Granbery, v. 8, p. 5, 2010.

MIGUEL, P. A. C. (Org.). Merchandising - Diferencial competitivo na otimização de resultados.Metodologia de pesquisa em engenharia de produção e gestão de operações. 2. ed. Rio de Janeiro: Elsevier, 2010.

OLIVEIRA, L. S; SANTOS, T. G. Merchandising - Diferencial competitivo na otimização de resultados. In: III ENCONTRO CIENTÍFICO E SIMPÓSIO DE EDUCAÇÃO UNISALESIANO. 3., 2011, Lins/ SP.

Anais... Lins: Centro Universitário UNISALESIANO, 2011. Disponível em:

<http://www.unisalesiano.edu.br/simposio2011/publicado/artigo0054.pdf>. Acesso em: 14 de nov. 2017.

PINHEIRO, J. Da Iniciação Científica ao TCC: Uma Abordagem para os cursos de Tecnologia. Rio de Janeiro: Ciência Moderna, 2010.

SHIMP, A. T. Comunicação de marketing: integrando propaganda, promoção e outras formas de divulgação. Tradução Martha Malvezzi Leal. São Paulo: Cengage Learning, 2012.

SILVA, R.D.O.; PINHEIRO, V. S. O Visual Merchandising de Loja de Varejo de Moda1 Análise da Loja Colcci. In: XXIX CONGRESSO BRASILEIRO DE CIÊNCIAS DA COMUNICAÇÃO, 29., 2006, Brasília/DF. Anais... Brasília: Universidade de Brasília, 2006. Disponível em:

<http://www.intercom.org.br/papers/nacionais/2006/resumos/R0562-1.pdf>. Acesso em: 14 de nov. 2017. 